\title{
Diagrammatic self-energy approximations and the total particle number
}

\author{
Arno Schindlmayr, ${ }^{1, *}$ P. García-González, ${ }^{2}$ and R. W. Godby ${ }^{3}$ \\ ${ }^{1}$ Fritz-Haber-Institut der Max-Planck-Gesellschaft, Faradayweg 4-6, 14195 Berlin-Dahlem, Germany \\ ${ }^{2}$ Departamento de Física Fundamental, Universidad Nacional de Educación a Distancia, Apartado 60141, 28080 Madrid, Spain \\ ${ }^{3}$ Department of Physics, University of York, Heslington, York YO10 5DD, United Kingdom
}

(Received 17 May 2001; published 19 November 2001)

\begin{abstract}
There is increasing interest in many-body perturbation theory as a practical tool for the calculation of ground-state properties. As a consequence, unambiguous sum rules such as the conservation of particle number under the influence of the Coulomb interaction have acquired an importance that did not exist for calculations of excited-state properties. In this paper we obtain a rigorous, simple relation whose fulfilment guarantees particle-number conservation in a given diagrammatic self-energy approximation. Hedin's $G_{0} W_{0}$ approximation does not satisfy this relation and hence violates the particle-number sum rule. Very precise calculations for the homogeneous electron gas and a model inhomogeneous electron system allow the extent of the nonconservation to be estimated.
\end{abstract}

DOI: $10.1103 /$ PhysRevB.64.235106

PACS number(s): 71.45.Gm, 71.15.Qe

\section{INTRODUCTION}

Many-body perturbation theory is a powerful method for studying interacting electron systems, because the partial summation of self-energy diagrams allows an efficient and systematically converging description of the dominant scattering mechanisms. ${ }^{1}$ In solid-state physics, Hedin's $G W$ approximation ${ }^{2}$ includes dynamic screening in the randomphase approximation and has been applied with great success to a large range of materials. ${ }^{3}$ While calculations have long focused on electronic excitations, such as band structures, ${ }^{4,5}$ that are not normally accessible by variational mean-field schemes, there is now increasing interest in using many-body perturbation theory also to obtain ground-state properties like the charge density ${ }^{6}$ or the total energy ${ }^{7-10}$ in order to circumvent well-known limitations of standard approximations in density-functional theory. ${ }^{11}$ Unlike the calculation of excited states, which are given immediately by the pole structure of the spectral function, this generally requires a multidimensional integration over the hole part of the Green function, as in Galitskii and Migdal's expression for the total energy. ${ }^{12}$ As a consequence, sum rules that could hitherto be ignored have gained new prominence. The most important of these is the conservation of particle number, i.e., the requirement that the integral

$$
N=\frac{1}{2 \pi i} \sum_{\sigma} \int d^{3} r \int d \omega G_{\sigma \sigma}(\mathbf{r}, \mathbf{r} ; \omega) e^{i \omega \eta}
$$

over the diagonal elements of the Green function $G$ equals the true number of electrons. Here $\sigma$ denotes the spin variable and $\eta$ is a positive infinitesimal that forces the frequency contour to be closed across the upper complex half plane.

In a seminal paper Baym and Kadanoff ${ }^{13}$ investigated the evolution of nonequilibrium Green functions and derived a set of symmetry relations for diagrammatic many-body approximations that guarantee the overall conservation of particle number, total energy, and momentum under timedependent external perturbations. Baym ${ }^{14}$ later showed that a self-energy satisfying all of these relations can be represented as the derivative $\Sigma=\delta \Phi / \delta G$ of a generating functional $\Phi$, and that in this case the Green function obtained self-consistently from Dyson's equation ${ }^{15}$ moreover yields the exact particle number. In particular, this applies to the fully self-consistent $G W$ approximation, in which both the Green function $G$ and the screened Coulomb interaction $W$ are dressed by self-energy insertions in accordance with a self-consistent solution of Dyson's equation. ${ }^{13}$ However, it has since become clear that $\Phi$ derivability is a sufficient but not a necessary requirement for the fulfilment of the particlenumber sum rule. For instance, the partially self-consistent $G W_{0}$ approximation, in which only the Green function is updated self-consistently but the screened Coulomb interaction remains undressed, is not $\Phi$ derivable but nevertheless produces the exact particle number. ${ }^{16}$ On the other hand, without self-consistency even in the Green function, the particle number is not, in general, given correctly, ${ }^{17}$ but this computationally efficient $G_{0} W_{0}$ approach still remains the preferred method for most practical applications.

More complicated self-energy expressions like the cumulant expansion ${ }^{18}$ or the $T$ matrix ${ }^{19}$ have already been successfully applied to solids, leading to an improved description of satellite resonances. Like the $G W$ approximation, these schemes are typically implemented without full selfconsistency and are hence not $\Phi$ derivable. In order to avoid expensive numerical tests in such situations, it would be desirable to have clear diagrammatic criteria for the fulfilment of the particle-number sum rule that could be checked a priori without actual calculations.

Unfortunately, Baym's proof, which is based on Luttinger's examination of the exact theory ${ }^{20}$ and determines the volume of the Fermi sea directly, cannot easily be extended, because it relies explicitly on the existence of the generating functional $\Phi$. We therefore take a different approach by describing the switching on of the Coulomb potential as a timedependent process that connects the noninteracting and the corresponding interacting electron system on a finite time scale. In this way we can examine the differential conservation laws and deduce a diagrammatic symmetry relation for 
particle-number conservation before taking the adiabatic limit. The theoretical framework is developed in Sec. II. The non-self-consistent $G_{0} W_{0}$ approximation, which violates this symmetry relation and does not conserve the particle number when the interaction is switched on, deserves special attention owing to its pre-eminent role in practical implementations. In Sec. III we therefore present very precise numerical calculations of the particle number for the homogeneous electron gas and a model inhomogeneous system in order to assess the quantitative deviation. Finally, in Sec. IV we summarize our conclusions. Atomic units are used throughout.

\section{PARTICLE-NUMBER CONSERVATION}

In order to connect the interacting electron system with the corresponding noninteracting system, whose properties are supposedly known exactly, we consider the Hamiltonian

$$
\hat{H}(t)=\hat{H}_{0}+e^{-\epsilon|t|} \hat{H}_{1} .
$$

The one-body part $\hat{H}_{0}$ contains the kinetic energy as well as the external potential $V_{\text {ext }}$, and the Coulomb interaction $\hat{H}_{1}$ is switched on exponentially with $\epsilon>0$. At large times, both in the past and in the future, the Hamiltonian reduces to $\hat{H}_{0}$, which constitutes a solvable problem. The noninteracting Green function $G_{0}$ is readily constructed from the solutions of the single-particle Schrödinger equation and yields the correct number of particles. On the other hand, at $t=0$ the full Coulomb interaction is effective, and the Green function is defined as ${ }^{1}$

$$
G\left(x, x^{\prime}\right)=-i \frac{\left\langle\Psi\left|T\left[\hat{\psi}(x) \hat{\psi}^{\dagger}\left(x^{\prime}\right)\right]\right| \Psi\right\rangle}{\langle\Psi \mid \Psi\rangle}
$$

where the shorthand notation $x \equiv(\mathbf{r}, \sigma, t)$ indicates a set of spatial, spin, and temporal coordinates, $|\Psi\rangle$ denotes the ground-state wave function of the interacting electron system in the Heisenberg picture, and $T$ is Wick's time-ordering operator that rearranges the subsequent symbols in ascending order from right to left with a sign change for every pair commutation. Furthermore, $\hat{\psi}^{\dagger}\left(x^{\prime}\right)$ and $\hat{\psi}(x)$ represent the electron creation and annihilation operator in the Heisenberg picture, respectively.

The unknown many-body wave function $|\Psi\rangle$ evolves from the noninteracting ground state $\left|\Psi_{0}\right\rangle$ and can formally be expressed as $|\Psi\rangle=\hat{U}_{\epsilon}(0,-\infty)\left|\Psi_{0}\right\rangle$, where $^{1}$

$$
\begin{aligned}
\hat{U}_{\epsilon}\left(t, t^{\prime}\right)= & \sum_{\nu=0}^{\infty} \frac{(-i)^{\nu}}{\nu !} \int_{t^{\prime}}^{t} d t_{1} \cdots \int_{t^{\prime}}^{t} d t_{\nu} e^{-\epsilon\left(\left|t_{1}\right|+\cdots+\left|t_{\nu}\right|\right)} \\
& \times T\left[\hat{H}_{1}\left(t_{1}\right) \cdots \hat{H}_{1}\left(t_{\nu}\right)\right]
\end{aligned}
$$

represents the time-development operator. With this definition the Green function may be rewritten as

$$
G\left(x, x^{\prime}\right)=-i \frac{\left\langle\Psi_{0}\left|T\left[\hat{S}_{\epsilon} \hat{\psi}(x) \hat{\psi}^{\dagger}\left(x^{\prime}\right)\right]\right| \Psi_{0}\right\rangle}{\left\langle\Psi_{0}\left|\hat{S}_{\epsilon}\right| \Psi_{0}\right\rangle}
$$

with $\hat{S}_{\epsilon}=\hat{U}_{\epsilon}(\infty,-\infty)$. At this stage the Gell-Mann and Low theorem ${ }^{21}$ asserts that it is, in general, permissible to take the adiabatic limit $\epsilon \rightarrow 0$. However, in the following we continue to perform a time-dependent perturbation analysis for finite $\epsilon$ and only take the adiabatic limit after establishing the conservation criteria that apply during the transition. The timeordered products in Eq. (5) may be evaluated in the usual way by invoking Wick's theorem, ${ }^{22}$ because the exponentials are scalar functions and commute with the field operators. Hence the perturbative treatment generates the standard series of connected and topologically distinct Feynman diagrams ${ }^{23}$ made up of the noninteracting Green function $G_{0}$ and the two-body Coulomb potential, but the latter now acquires an additional prefactor and is given by

$$
v\left(x, x^{\prime}\right)=\frac{e^{-\epsilon|t|}}{\left|\mathbf{r}-\mathbf{r}^{\prime}\right|} \delta\left(t-t^{\prime}\right) .
$$

The formal identity of the perturbation expansion in the time-dependent and the adiabatic, time-independent case is a crucial result that forms the basis of our discussion in this section.

For an analysis of the conservation properties we now follow Ref. 13 and write the perturbation series as

$$
\begin{aligned}
& \int G_{0}^{-1}\left(x, x_{1}\right) G\left(x_{1}, x^{\prime}\right) d x_{1} \\
& =\delta\left(x-x^{\prime}\right)-i \int v\left(x, x_{1}\right) G_{2}\left(x, x_{1} ; x^{\prime}, x_{1}^{+}\right) d x_{1},
\end{aligned}
$$

invoking the two-particle Green function $G_{2}$. The superscript $x^{+}$indicates that a positive infinitesimal is added to the time variable to ensure the proper ordering. An equivalent form is the adjoint equation of motion

$$
\begin{aligned}
& \int G\left(x, x_{1}\right) G_{0}^{-1}\left(x_{1}, x^{\prime}\right) d x_{1} \\
& \quad=\delta\left(x-x^{\prime}\right)-i \int G_{2}\left(x, x_{1} ; x^{\prime}, x_{1}^{+}\right) v\left(x^{\prime}, x_{1}\right) d x_{1} .
\end{aligned}
$$

The inverse noninteracting Green function is identical to the operator

$$
\begin{aligned}
G_{0}^{-1}\left(x, x^{\prime}\right) & =\left(i \frac{\partial}{\partial t}+\frac{1}{2} \nabla^{2}-V_{\mathrm{ext}}(\mathbf{r})\right) \delta\left(x-x^{\prime}\right) \\
& =\left(-i \frac{\partial}{\partial t^{\prime}}+\frac{1}{2} \nabla^{\prime 2}-V_{\mathrm{ext}}\left(\mathbf{r}^{\prime}\right)\right) \delta\left(x-x^{\prime}\right),
\end{aligned}
$$

so that after substracting Eq. (8) from Eq. (7) we obtain 


$$
\begin{aligned}
{\left[i \left(\frac{\partial}{\partial t}+\right.\right.} & \left.\left.\frac{\partial}{\partial t^{\prime}}\right)+\frac{1}{2}\left(\nabla+\nabla^{\prime}\right) \cdot\left(\nabla-\nabla^{\prime}\right)\right] G\left(x, x^{\prime}\right) \\
= & {\left[V_{\mathrm{ext}}(\mathbf{r})-V_{\mathrm{ext}}\left(\mathbf{r}^{\prime}\right)\right] G\left(x, x^{\prime}\right)-i } \\
& \times \int\left[v\left(x, x_{1}\right)-v\left(x^{\prime}, x_{1}\right)\right] G_{2}\left(x, x_{1} ; x^{\prime}, x_{1}^{+}\right) d x_{1} .
\end{aligned}
$$

When we set $x^{\prime}=x^{+}$, the terms on the right-hand side cancel, while the left-hand side reduces to the differential conservation law for the particle number

$$
\frac{\partial n(\mathbf{r}, t)}{\partial t}+\nabla \cdot \mathbf{j}(\mathbf{r}, t)=0
$$

with the electron density $n(\mathbf{r}, t)=-i \Sigma_{\sigma} G\left(x, x^{+}\right)$and current $\mathbf{j}(\mathbf{r}, t)=-\frac{1}{2} \Sigma_{\sigma}\left[\left(\nabla-\nabla^{\prime}\right) G\left(x, x^{\prime}\right)\right]_{x^{\prime}=x^{+}}$. Thus whenever Eqs. (7) and (8) are satisfied simultaneously, the total particle number is conserved while the interaction is switched on. This does not depend on the value of $\epsilon$ and, in particular, remains true in the adiabatic limit, which can now be taken, turning $G$ into the equilibrium Green function of the interacting electron system.

By multiplying Eqs. (7) and (8) with $G$ from the left and right, respectively, and then subtracting one from the other, their mutual consistency can be stated in the more convenient form,

$$
\begin{aligned}
\int & G\left(x, x_{2}\right) v\left(x_{2}, x_{1}\right) G_{2}\left(x_{2}, x_{1} ; x^{\prime}, x_{1}^{+}\right) d x_{1} d x_{2} \\
= & \int G_{2}\left(x, x_{1} ; x_{2}, x_{1}^{+}\right) v\left(x_{2}, x_{1}\right) G\left(x_{2}, x^{\prime}\right) d x_{1} d x_{2},
\end{aligned}
$$

that may easily be verified by visual inspection of a given diagrammatic approximation for the two-particle Green function. Evidently it is the same criterion as derived by Baym and Kadanoff ${ }^{13}$ for particle-number conservation under time-dependent external perturbations. This is no coincidence, of course, because the termwise cancellation of diagrams on the right-hand side of Eq. (10) is of purely topological origin and does not depend on the mathematical properties of the constituent propagators. Hence it is inconsequential whether, as in Refs. 13 and $14, G_{0}$ contains a time-dependent perturbation while the interaction is constant or, as in the physical situation considered here, the noninteracting Green function is invariant under temporal translations while the Coulomb potential instead acquires a timedependent prefactor.

As an example we now consider the $G W$ approximation. The self-energy, when applied with full self-consistency, is given by

$$
\Sigma\left(x, x^{\prime}\right)=i G\left(x, x^{\prime}\right) W\left(x^{+}, x^{\prime}\right),
$$

where the screened Coulomb interaction $W$ takes the mathematical form of the random-phase approximation but is evaluated using the dressed Green function self-consistently (a)

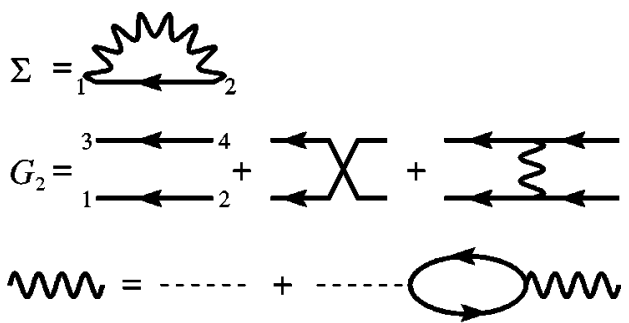

(b)

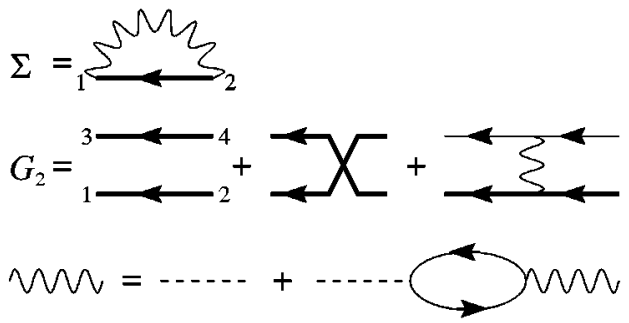

(c)

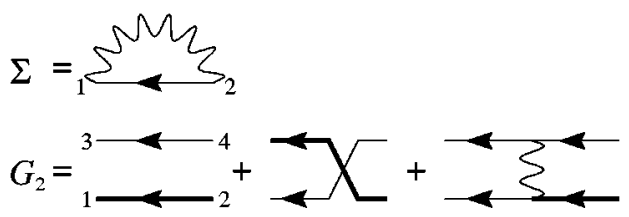

FIG. 1. Diagrammatic representation of the self-energy $\Sigma\left(x_{1}, x_{2}\right)$ and the corresponding two-particle Green function $G_{2}\left(x_{1}, x_{3} ; x_{2}, x_{4}\right)$ in (a) the fully self-consistent $G W$ approximation, (b) the partially self-consistent $G W_{0}$ approximation, and (c) the non-self-consistent $G_{0} W_{0}$ approximation.

derived from Dyson's equation. The diagrammatic representation of $\Sigma$ is shown in Fig. 1(a). The corresponding twoparticle Green function is obtained by comparing Dyson's equation with the equation of motion (7), which yields the identity

$$
\begin{aligned}
& -i \int v\left(x, x_{1}\right) G_{2}\left(x, x_{1} ; x^{\prime}, x_{1}^{+}\right) d x_{1} \\
& \quad=V_{H}(x) G\left(x, x^{\prime}\right)+\int \Sigma\left(x, x_{1}\right) G\left(x_{1}, x^{\prime}\right) d x_{1},
\end{aligned}
$$

where $V_{H}(x)=-i \int v\left(x, x_{1}\right) G\left(x_{1}, x_{1}^{+}\right) d x_{1}$ indicates the Hartree potential. The two-particle Green function corresponding to the $G W$ approximation for the self-energy is also displayed in Fig. 1(a). It is easily seen that it satisfies the symmetry relation (12), which is essentially a horizontal leftright symmetry for the building blocks of $G_{2}$, and hence conserves the total particle number when the Coulomb interaction is switched on. Of course, this result also follows from the existence of the generating functional $\Phi .^{14}$

The partially self-consistent $G W_{0}$ approximation

$$
\Sigma\left(x, x^{\prime}\right)=i G\left(x, x^{\prime}\right) W_{0}\left(x^{+}, x^{\prime}\right),
$$

in which the screened Coulomb interaction $W_{0}$ is evaluated with the noninteracting Green function $G_{0}$, is not $\Phi$ derivable, which would require an additional vertical mirror symmetry $G_{2}\left(x_{1}, x_{3} ; x_{2}, x_{4}\right)=G_{2}\left(x_{3}, x_{1} ; x_{4}, x_{2}\right)$ in the diagrammatic structure of the two-particle Green function that has 
been lost in the transition from full to partial selfconsistency. Nevertheless, $G_{2}$, shown in Fig. 1(b), still obeys the consistency relation (12) and hence guarantees the correct total particle number, as previously confirmed by explicit integration of the spectral function. ${ }^{16}$ In contrast, the non-self-consistent $G_{0} W_{0}$ approximation

$$
\Sigma\left(x, x^{\prime}\right)=i G_{0}\left(x, x^{\prime}\right) W_{0}\left(x^{+}, x^{\prime}\right)
$$

leads to a two-particle Green function with lower internal symmetry, displayed in Fig. 1(c), that no longer satisfies Eq. (12), implying an incorrect total particle number. The quantitative deviation is investigated in the following section. In a similar manner, the conservation properties of other diagrammatic self-energy approximations are easily established by an inspection of the underlying two-particle Green function.

\section{NUMERICAL RESULTS}

In the previous section we proved that the $G W$ and $G W_{0}$ approximations conserve the particle number for an arbitrary electron system when the Coulomb interaction is switched on, in contrast to $G_{0} W_{0}$. This, coupled with their superior performance in ground-state total-energy calculations, 7,10 might be thought to suggest that the $G_{0} W_{0}$ approach is useless if one is interested in ground-state properties. However, a many-body calculation at only the $G_{0} W_{0}$ level is already sufficient to correct typical limitations of mean-field densityfunctional theories, such as their inaccuracy in highly inhomogeneous systems or their failure to describe van der Waals forces. ${ }^{24}$ Moreover, the Green function arising from a $G_{0} W_{0}$ calculation may be used as input in the variational Luttinger and Ward functional, ${ }^{25}$ and prospective calculations suggest that this is an excellent approach for calculating total energies. ${ }^{26}$ Since these methods are more amenable to applications in complex systems than the fully or partially selfconsistent $G W$ approximations, it is important to determine whether the underlying violation of the particle-number sum rule in the $G_{0} W_{0}$ framework is small enough to be safely ignored.

There are some indications that such an error is indeed fairly small for the homogeneous electron gas at metallic densities, ${ }^{16}$ a Hubbard model system, ${ }^{17}$ and typical semiconductors. ${ }^{6}$ Here, bearing in mind that many-body total-energy calculations are intended to be used in extreme situations where standard implementations of densityfunctional theory fail, we present numerical results for thin jellium slabs, whose most relevant feature is the strong inhomogeneity of the electron-density profile, as well as for the homogeneous electron gas over a wide range of densities.

Our concern is the evaluation of the particle-number difference,

$$
\delta N=\frac{-i}{\pi} \int_{-\infty}^{+\infty} d \omega \operatorname{tr}\left[G(\omega)-G_{0}(\omega)\right]
$$

where tr denotes the spatial trace (we omit the explicit spatial variables for clarity and also consider only spin-unpolarized systems). Since both $G$ and $G_{0}$ behave as $1 / \omega$ for large frequencies, we can apply Cauchy's theorem and write Eq. (17) alternatively as

$$
\delta N=\frac{1}{\pi} \int_{-\infty}^{+\infty} d \omega \operatorname{tr}\left[G(\mu+i \omega)-G_{0}\left(\mu_{0}+i \omega\right)\right],
$$

where $\mu$ and $\mu_{0}$ are the chemical potentials of the interacting and the noninteracting system, respectively, which correspond, by definition, to the position of the pole of the Green function at the Fermi surface. As the characteristic sharp structure of $G(\omega)$ (quasiparticle peaks and satellites) does not appear in the analytic continuation $G(\mu+i \omega)$, Eq. (18) is preferred for numerical integration. We hence follow some of the ideas suggested by Rojas et al. ${ }^{27}$ and work exclusively in an imaginary time and frequency representation. An accurate evaluation of Eq. (18) furthermore requires a treatment of the high-frequency tails of $G$, which can be done easily with the numerical procedures described in Ref. 28.

For the homogeneous electron gas, an analytic expression exists for the noninteracting Green function $G_{0}(r, i \tau)$ in real space and imaginary time, ${ }^{29}$ while the screened Coulomb interaction $W_{0}(k, i \omega)$ in the random-phase approximation is given analytically in reciprocal space by the dynamic Lindhard function. ${ }^{30}$ The evaluation of the self-energy according to $\Sigma(r, i \tau)=i G_{0}(r, i \tau) W_{0}(r, i \tau)$ therefore only requires the numerical Fourier transform $W_{0}(k, i \omega)$ $\rightarrow W_{0}(r, i \tau)$. It is this largely analytic approach that makes the present calculation especially precise.

At this stage we remark that the self-energy given by Eq. (16) has the same analytic structure as the underlying Green function $G_{0}$, i.e., the poles of $\Sigma(\omega)$ are located in the upper (lower) complex half-plane for energies smaller (larger) than $\mu_{0}=\frac{1}{2} k_{\mathrm{F}}^{2}$, where $k_{\mathrm{F}}$ denotes the Fermi wave vector. As a consequence, an inconsistency arises because the true selfenergy should have a polar structure identical to the interacting Green function with the chemical potential $\mu$. The selfenergy must therefore be appropriately shifted along the real frequency axis. In the imaginary time/frequency representation, this shift is automatically included in the backward transform

$$
\Sigma(\mu+i \omega)=-i \int_{-\infty}^{+\infty} d \tau \Sigma(i \tau) e^{-i \omega \tau} .
$$

The calculation of $\Sigma(\mu+i \omega)$, therefore, does not require an advance knowledge of $\mu$, which can now be obtained from the relation $\mu=\mu_{0}+\Sigma\left(k_{\mathrm{F}}, \mu\right)$.

Finally, the interacting Green function is calculated in reciprocal space according to

$$
G(k, \mu+i \omega)=\frac{1}{i \omega-\frac{1}{2} k^{2}-\Sigma(k, \mu+i \omega)+\mu} .
$$

In the same representation, the noninteracting Green function is given by

$$
G_{0}\left(k, \mu_{0}+i \omega\right)=\frac{1}{i \omega-\frac{1}{2} k^{2}+\mu_{0}}
$$




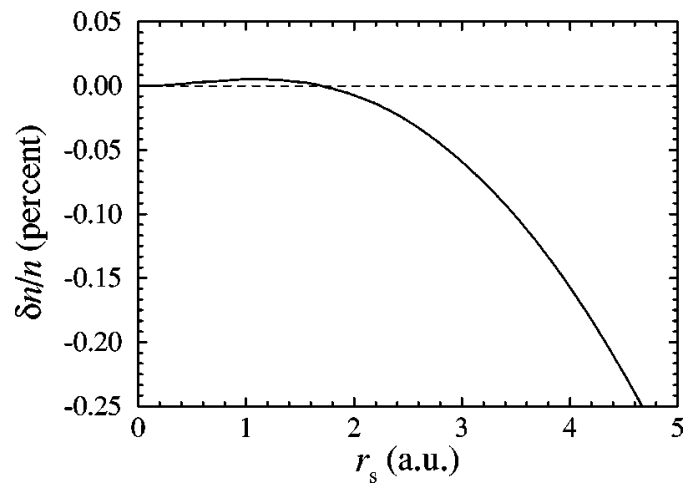

FIG. 2. Violation of the particle-number sum rule for the homogeneous electron gas in the $G_{0} W_{0}$ approximation. The relative error in the density is always negative and of the order of $0.1 \%$ in the range of metallic densities.

so that the density variation is readily obtained from

$$
\delta n=\int \frac{d^{3} k}{(2 \pi)^{3}} \int_{-\infty}^{+\infty} \frac{d \omega}{\pi}\left[G(k, \mu+i \omega)-G_{0}\left(k, \mu_{0}+i \omega\right)\right] .
$$

In Fig. 2 the relative devation $\delta n / n_{0}$ from the exact density is displayed as a function of the Wigner-Seitz radius $r_{\mathrm{s}}$. In the high-density region $r_{\mathrm{s}}<1.8$ the particle number is slightly overestimated $(<0.01 \%)$, while it is underestimated for lower densities. In the range of metallic densities this underestimation is of the order of $0.1 \%$, but the error becomes increasingly important in the dilute limit $(-1.7 \%$ for $r_{\mathrm{s}}=10$ and $-6.1 \%$ for $r_{\mathrm{s}}=20$ ).

As pointed out above, it is also of interest to investigate the error resulting from the $G_{0} W_{0}$ method in the total number of particles for a strongly inhomogeneous system. The model we have chosen is a thin jellium slab with a background density $n_{0}=\left(\frac{4}{3} \pi r_{\mathrm{s}}^{3}\right)^{-1}$ and width $L$. The slab is bounded by two infinite planar walls, so that, if charge neutrality is assumed, the system is fully characterized by the lengths $r_{\mathrm{s}}$ and $L$. In this case, $G_{0}$ corresponds to the KohnSham system obtained self-consistently with the localdensity approximation (LDA) for the exchange-correlation potential $V_{\mathrm{xc}}$, as is typically done in practical ab initio calculations.

With $z$ chosen as the coordinate perpendicular to the planar walls, the translational symmetry of the system in the $x y$ plane allows an efficient semianalytic evaluation of the relevant propagators. The screened Coulomb interaction is given by $W_{0}=\epsilon_{0}^{-1} v$, where $\epsilon_{0}$ denotes the dielectric function in the random-phase approximation. The latter is calculated as $\epsilon_{0}(k, i \omega)_{\alpha \beta}$ in the basis $\zeta_{\alpha}(z) \exp (i \mathbf{k} \cdot \boldsymbol{\rho}) / \sqrt{S}$. Here $\zeta_{\alpha}(z)$ is a set of cosine functions, $\mathbf{k}=\left(k_{x}, k_{y}\right)$ and $\boldsymbol{\rho}=(x, y)$ denote the two-dimensional momentum and the position vector in the $x y$ plane, respectively, and $S$ is the slab surface. The matrix elements can be calculated analytically in terms of the scalar products $\left\langle\zeta_{\alpha} \phi_{n} \mid \phi_{m}\right\rangle,{ }^{24,31}$ where $\phi_{n}(z) \exp (i \mathbf{k} \cdot \boldsymbol{\rho}) / \sqrt{S}$ are the single-particle eigenstates of the Kohn-Sham Hamiltonian $h_{\mathrm{KS}}$. The matrix elements $v(k)_{\alpha \beta}$ of the Coulomb potential are likewise obtained analytically. The screened

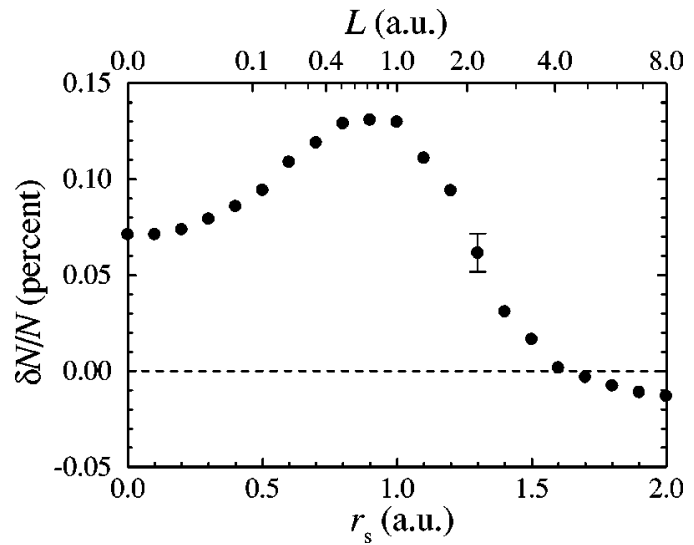

FIG. 3. Relative violation of particle number in the $G_{0} W_{0}$ approximation for thin jellium slabs of fixed 2D density $n^{2 \mathrm{D}}=3 / 4 \pi$ as a function of their thickness $L$ (and the corresponding 3D density parameter $r_{\mathrm{s}}$ ). A typical error bar is reported.

Coulomb interaction is then easily calculated by a matrix inversion for each value of $k$, and the real-space representation is given by expanding

$$
\begin{aligned}
W_{0}\left(\rho, z, z^{\prime} ; i \tau\right)= & i \sum_{\alpha, \beta} \int \frac{d^{2} k}{(2 \pi)^{2}} \int_{-\infty}^{+\infty} \frac{d \omega}{2 \pi} e^{i(\omega \tau+\mathbf{k} \cdot \boldsymbol{\rho})} \\
& \times \zeta_{\alpha}(z) \zeta_{\beta}\left(z^{\prime}\right) W_{0}(k, i \omega)_{\alpha \beta} .
\end{aligned}
$$

The Green function $G_{0}\left(\rho, z, z^{\prime} ; i \tau\right)$ is readily calculated from the Kohn-Sham eigenstates, and by employing Eq. (16) we obtain the self-energy in real space and imaginary time as well as, eventually, its representation $\Sigma(k, \mu+i \omega)_{n m}$ in the Kohn-Sham basis set. The presence of infinite confining walls implies a quick convergence with respect to the number of cosine and Kohn-Sham wave functions used in the calculation. The convergence is further accelerated by the analytic treatment of the asymptotic time and frequency tails of all operators.

The Green function is calculated in the basis of KohnSham eigenstates according to

$$
G(k, \mu+i \omega)=\left[i \omega-h_{\mathrm{KS}}(k)-\Sigma(k, \mu+i \omega) .+V_{\mathrm{xc}}(k)+\mu\right]^{-1}
$$

by a matrix inversion in the indices $\mathrm{nm}$. Finally, the variation of the number of particles per surface unit is given by

$$
\begin{aligned}
\frac{\delta N}{S}= & \sum_{m} \int \frac{d^{2} k}{(2 \pi)^{2}} \int_{-\infty}^{+\infty} \frac{d \omega}{\pi}\left[G(k, \mu+i \omega)_{m m}\right. \\
& \left.-G_{0}\left(k, \mu_{0}+i \omega\right)_{m m}\right],
\end{aligned}
$$

where we have used the invariance of the trace with respect to any wave-function representation.

In Fig. 3 we plot the relative deviation of the particle number $\delta N / N$ in the $G_{0} W_{0}$ approximation for several configurations of the model system, keeping the exact number of particles per surface unit $n^{2 \mathrm{D}}=n_{0} L=L /\left(\frac{4}{3} \pi r_{\mathrm{s}}^{3}\right)$ constant. The limit $L \rightarrow 0$ thus corresponds to a two-dimensional (2D) homogeneous electron gas with density $n^{2 \mathrm{D}}$. Over the wide 
variation of the degree of homogeneity shown in the figure, it is seen that $\delta N / N$ remains of similar magnitude as in the homogeneous case $(\leq 0.2 \%)$. This observation remains true for other $2 \mathrm{D}$ densities inside the range $[0.1,1]$.

\section{CONCLUSIONS}

In this paper we have rigorously obtained a general criterion which allows, by simple inspection, to verify whether a diagrammatic self-energy approximation satisfies the particle-number sum rule for an interacting electron system. As an application, we have demonstrated that the so-called $G_{0} W_{0}$ method does not yield the correct particle number, generalizing the conclusions of a previous analytic study for a Hubbard model Hamiltonian defined only on a discrete lattice. ${ }^{17}$ Thus this limitation of the $G_{0} W_{0}$ approximation has been fully confirmed for arbitrary electron systems. By per-

*Electronic address: schindlmayr@fhi-berlin.mpg.de

${ }^{1}$ A.L. Fetter and J.D. Walecka, Quantum Theory of Many-Particle Systems (McGraw-Hill, New York, 1971).

${ }^{2}$ L. Hedin, Phys. Rev. 139, A796 (1965).

${ }^{3}$ F. Aryasetiawan and O. Gunnarsson, Rep. Prog. Phys. 61, 237 (1998).

${ }^{4}$ M.S. Hybertsen and S.G. Louie, Phys. Rev. Lett. 55, 1418 (1985); Phys. Rev. B 34, 5390 (1986).

${ }^{5}$ R.W. Godby, M. Schlüter, and L.J. Sham, Phys. Rev. Lett. 56, 2415 (1986); Phys. Rev. B 35, 4170 (1987).

${ }^{6}$ M.M. Rieger and R.W. Godby, Phys. Rev. B 58, 1343 (1998).

${ }^{7}$ B. Holm, Phys. Rev. Lett. 83, 788 (1999).

${ }^{8}$ B. Holm and F. Aryasetiawan, Phys. Rev. B 62, 4858 (2000).

${ }^{9}$ P. Sánchez-Friera and R.W. Godby, Phys. Rev. Lett. 85, 5611 (2000)

${ }^{10}$ P. García-González and R.W. Godby, Phys. Rev. B 63, 075112 (2001).

${ }^{11}$ P. Hohenberg and W. Kohn, Phys. Rev. 136, B864 (1964); W. Kohn, and L.J. Sham, ibid. 140, A1133 (1965).

${ }^{12}$ V.M. Galitskii and A.B. Migdal, Zh. Éksp. Teor. Fiz. 34, 139 (1958) [Sov. Phys. JETP 7, 96 (1958)].

${ }^{13}$ G. Baym and L.P. Kadanoff, Phys. Rev. 124, 287 (1961).

${ }^{14}$ G. Baym, Phys. Rev. 127, 1391 (1962). forming a very precise integration of the spectral function, we have furthermore calculated the size of the error in the $G_{0} W_{0}$ particle number in two simple, but very distinct, families of electron systems. The error becomes large only outside the range of densities of physical interest.

\section{ACKNOWLEDGMENTS}

The authors thank Professor C.-O. Almbladh and Dr. J. E. Alvarellos for a thorough reading of the manuscript and for valuable discussions. This work was funded in part by the EU through the NANOPHASE Research Training Network (Contract No. HPRN-CT-2000-00167), the Spanish Education Ministry DGESIC Grant No. PB97-1223-C02-02, and by the Deutscher Akademischer Austauschdienst and the British Council under the British-German Academic Research Collaboration (ARC) program.

${ }^{15}$ F.J. Dyson, Phys. Rev. 75, 486 (1949); 75, 1736 (1949).

${ }^{16}$ B. Holm, Ph.D. thesis, Lund University, 1997.

${ }^{17}$ A. Schindlmayr, Phys. Rev. B 56, 3528 (1997).

${ }^{18}$ F. Aryasetiawan, L. Hedin, and K. Karlsson, Phys. Rev. Lett. 77, 2268 (1996).

${ }^{19}$ M. Springer, F. Aryasetiawan, and K. Karlsson, Phys. Rev. Lett. 80, 2389 (1998)

${ }^{20}$ J.M. Luttinger, Phys. Rev. 119, 1153 (1960).

${ }^{21}$ M. Gell-Mann and F. Low, Phys. Rev. 84, 350 (1951).

${ }^{22}$ G.C. Wick, Phys. Rev. 80, 268 (1950).

${ }^{23}$ R.P. Feynman, Phys. Rev. 76, 749 (1949); 76, 769 (1949).

${ }^{24}$ P. García-González and R. W. Godby (unpublished).

${ }^{25}$ J.M. Luttinger and J.C. Ward, Phys. Rev. 118, 1417 (1960).

${ }^{26}$ C.-O. Almbladh, U. von Barth, and R. van Leeuwen, Int. J. Mod. Phys. B 13, 535 (1999).

${ }^{27}$ H.N. Rojas, R.W. Godby, and R.J. Needs, Phys. Rev. Lett. 74, 1827 (1995).

${ }^{28}$ L. Steinbeck, A. Rubio, L. Reining, M. Torrent, I.D. White, and R.W. Godby, Comput. Phys. Commun. 125, 105 (2000).

${ }^{29}$ A. Schindlmayr, Phys. Rev. B 62, 12573 (2000).

${ }^{30}$ J. Lindhard, K. Dan. Vidensk. Selsk. Mat. Fys. Medd. 28, 8 (1954).

${ }^{31}$ A.G. Eguiluz, Phys. Rev. B 31, 3303 (1985); J.M. Pitarke and A.G. Eguiluz, ibid. 63, 045116 (2001). 